band moves down the column, and a buffer of suitable $p H$ is found by trial. On a given buffered column, no significant variation in rate of band movement of a particular dinitrophenyl amino-acid between different batches of gel is observed, and the difficulty involved in preparing a suitable gel does not arise. Using a single solvent in conjunction with columns buffered to different $p H$ values, the number of such acids which can be separated from each other is considerably greater than the number which can be separated in the same solvent with unbuffered columns.

The method of separation of mixtures of dinitrophenyl amino-acids I have used, which is based on these observations, differs somewhat from that given by Porter and Sanger ${ }^{3}$. Buffered chloroform and butanol-chloroform columns are employed for the initial separations. Subsequent separations are carried out using buffered columns and other solvents. Buffered 5 per cent propanolcyclohexane columns are useful for separating dinitrophenyl monoamino-acids from each other, and in general they bring about those separations that Sanger's ethanol-ligroin columns do. Thus on these columns dinitrophenylleucine can be separated from dinitrophenyl-valine and the latter from dinitrophenyl-alanine. The slower-moving dinitrophenyl amino-acids have characteristic rates on buffered ether columns and can be separated from each other in this way. The fastermoving acids have characteristic rates on buffered 33 per cent ether-ligroin columns, and it is probable that useful separations could also be brought about in this solvent.

The increasing use of buffered silica gel in partition chromatograms, for example, in the separation of fatty acids ${ }^{6}$ and penicillins', ${ }^{\prime}$, suggests that this type of column may be capable of very wide application. A fuller description of the present experiments will be given elsewhere. I am indebted to the Director of the Wool Industries Research Association for permission to publish this note.

\section{S. BLACKBURN}

Wool Industries Research Association,

Torridon, Headingley,

Leeds 6.

Dec. 22.

${ }^{1}$ Middlebrook, W. R., and Phillips, H., J. Soc. Dyers and Col., 57, 137 (1941).

'Sanger, F., Biochem. J., 39, 507 (1945).

${ }^{3}$ Porter, R. R., and Sanger, F., Biochem. J., 42, 287 (1948).

4 Phillips, D. M. P., and Stephen, J. M. L., Nature, 162, 152 (1948).

5 Consden, R., Gordon, A. H., Martin, A. J. P., and Synge, R. I. M., Biochem. J., 41, 596 (1947). Moyle, V., Baldwin, E., and Scarisbrick, R., Biochem. J., 43, 308
(1948).

'Levi, A. A., Biochem. J., 43, 257 (1948). - Boon, W. R., Calam, C. T., Gudgeon, H., and Levi, A. A., Biochem. J.,
43, 262 (1948).

\section{A Possible Nuclear Effect in Magnetic Cooling Experiments on Copper Salts}

Atrention has recently been directed by Gorter ${ }^{1}$ to the existence of a splitting of the energy-levels of paramagnetic salts due to the coupling between the electronic and nuclear magnetic moments. The level separation, which is of the order of $0.1 \mathrm{~cm} .^{-1}$, is seen in the hyperfine structure of the optical line spectrum. Now such a splitting ought also to lead to a Schottky specific heat anomaly at temperatures of the order of $0 \cdot 1^{\circ} \mathrm{K}$., that is, some $10^{4}$ times higher than in salts the paramagnetism of which is due only to the nuclei.

Of substances used in magnetic cooling experiments, it appears that the copper salts are almost unique in that copper possesses both electronic paramagnetism and a non-zero nuclear moment. The hyperfine structure of the optical are spectrum of copper has been observed by Ritschl ${ }^{2}$. If the electric crystalline field and the other interactions in the crystal be ignored, then Ritschl's value of $0.022 \mathrm{~cm}^{-1}$ $\left(0.031^{\circ} \mathrm{K}\right.$.) for the splitting-factor of the ${ }^{3} d^{9}{ }^{2} D_{5 / 2}$ state (the ground-state in the crystal before the introduction of the crystalline field ${ }^{3}$ ) would lead to a specific heat of the order of $(0.031)^{2} R / T^{2} \sim 10^{-3} R / T^{2}$ per gm.-ion. Very recently a hyperfine structure has been observed by Penrose ${ }^{4}$ in the paramagnetic resonance lines in very dilute copper salts, from which it appears that the splitting in the crystal is similar in order of magnitude to that observed by Ritschl for the vapour, so that the above estimate of the size of the specific heat should not be seriously in error. From a study of this resonance line pattern it should be possible to obtain a better estimate of the magnitude of the nuclear specific heat; but meanwhile it may be of interest to see what experimental evidence there is for its existence.

The specific heat and susceptibility of copper potassium sulphate below $1^{\circ} \mathrm{K}$. have been measured by de Klerk ${ }^{5}$ and by $\mathrm{me}^{6}$; I find that $C=6.1 \times$ $10^{-4} R / T^{2}$, a value which is some five times larger than one would expect from dipole-dipole interaction. The excess has been ascribed by Opechowski ${ }^{7}$ to anisotropic exchange; but his theory gives no indication of the magnitude of the effect expected from this cause. It is possible that at least part of the excess specific heat may be due instead to the electronicnuclear coupling under discussion, and it will be seen that the observed specific heat is in agreement as to order of magnitude with this idea. For copper sulphate, Ashmead ${ }^{8}$ has found two maxima in the gamma-ray specific heat $C^{*}$, and it is probable that there are two corresponding maxima in the true specific heat. The upper maximum at about $1^{\circ} \mathrm{K}$. can be understood as corresponding to the removal of the electronic degeneracy by exchange effects ${ }^{9}$, but it has always been difficult to understand how both anomalies could be due to the electronic system. According to the present suggestion, the lower maximum might be caused by electronic-nuclear coupling, provided that this is not itself quenched by exchange effects.

Note added in proof.--Since this letter was written, Prof. Gorter has informed me that specific heat measurements on diluted copper potassium sulphate have been made by De Vrijer and theoretical calculations carried out by Broer, which, in general, confirm the views expressed in this letter.

\section{G. B. Garretr}

Royal Society Mond Laboratory,

\section{Cambridge.}

March 26.

1 Gorter, Physica, 14, 504 (1948).

'2 Ritschl, R.; Z. Physik, 79, 1 (1932).

${ }^{8}$ Polder, Physica, 9, 709 (1942).

- Penrose (private communication).

${ }^{5}$ de Klerk, Physica, 12, 513 (1946).

- Garrett, Cérémonies Langevin - Perrin, Paris. 1948 (in the press).

' Opechowski, Physica, 14, 236 (1946).

${ }^{8}$ Ashmead, Nature, 143, 853 (1939).

- Pryce, Nature, 162, 538 (1948). 